\title{
Minimum inhibitory concentration-guided antimicrobial therapy - the Achilles heel in the antimicrobial stewardship agenda
}

Antimicrobial stewardship (AMS) plays a pivotal role in the fight against antimicrobial resistance. A fundamental tenet of AMS is dose optimisation, whereby appropriate usage ensures clinical benefit. Dose optimisation in practice requires specific knowledge of the agent, the patient and the pathogen involved. At present, efforts to dose-optimise are based largely on knowledge of the agent and patient, with less emphasis on the pathogen. Detailed knowledge of the pathogen and its susceptibility to a particular agent is seldom available or considered in dose-optimisation strategies. I wish to highlight the minimum inhibitory concentration (MIC) as a crucial determinant in doseoptimisation strategies at the bedside and demonstrate the importance of MIC-guided antimicrobial therapy.

Antimicrobial susceptibility testing (AST) is a critical function of any microbiology laboratory, as it forms the basis upon which decisions on treatment of infectious diseases are made. The AST result provided on a laboratory report in the form of an S (susceptible), I (intermediate) or R (resistant) is derived from clinical breakpoints. Clinical breakpoints are in turn derived from analysis of the MIC value for a particular drug-bug combination. The MIC is defined as the lowest concentration of antibiotic required to inhibit visible growth of a micro-organism over a defined time interval. There is therefore a defined concentration of antimicrobial (MIC value) associated with the categorisation of an isolate as S, I or R. For example, an Escherichia coli isolate susceptible to ampicillin will have an MIC $\leq 8 \mu \mathrm{g} / \mathrm{mL}$ (as per European Committee on Antimicrobial Susceptibility Testing (EUCAST) clinical breakpoints $\left.{ }^{[1]}\right)$. These MIC values are not routinely reported, but rather the categorisation (S/I/R) only. The reason that MICs are seldom reported has more to do with the AST methodology than anything else. The gold standard for MIC testing is broth microdilution (BMD) or agar dilution, methods not practically feasible for a routine diagnostic laboratory. Hence routine AST results are based on alternative methods that have been validated against the gold standard, which then extrapolate to the MIC with a reported categorisation (S/I/R). The categorisation (S/I/R) highlights those agents that are more likely to achieve a successful outcome (i.e. agents reported as susceptible). However, antimicrobial resistance is a relative phenomenon, as illustrated by the following clinical scenario: empirical treatment of a particular infectious disease with good clinical response, only to subsequently find that the pathogen isolated is reported resistant to the chosen empirical agent. The explanation for this is that resistance is relative, and owing to a multitude of other factors, principally related to drug exposure (see below), the antimicrobial was active against the isolated pathogen. This gives rise to the $90 / 60$ rule in AST - $90 \%$ of patients treated with an antimicrobial that is reported as $\mathrm{S}$ will respond clinically, whereas only $60 \%$ of patients treated with an antimicrobial that is reported as $\mathrm{R}$ will respond clinically. ${ }^{[2]}$ The most important factor responsible for this observation is the well-described influence of pharmacokinetic-pharmacodynamic (Pk-Pd) target optimisation. ${ }^{[3]}$ This is illustrated by the example of an extended-spectrum betalactamase (ESBL)-producing E. coli in urine reported as R to cefepime, yet the clinical response is favourable despite this. The high renal elimination of unchanged drug means that even at a low dose $(250 \mathrm{mg})$ urinary concentrations of cefepime are $\sim 90-190 \mu \mathrm{g} / \mathrm{mL}$, well in excess of the clinical breakpoint of $1 \mu \mathrm{g} / \mathrm{mL}$ (EUCAST breakpoint). ${ }^{[1,4]}$ In the era of multidrug resistance the reporting of isolates as simply ' $R$ ' when based on an MIC they may be therapeutic options results in limited antimicrobial choices, reliance on broad-spectrum agents and limited ability to tailor antimicrobial therapy to the individual.

Antimicrobial efficacy can be correlated with its particular Pk-Pd parameter. There are three defined Pk-Pd parameters: (i) percentage duration of time that free-drug concentration exceeds the MIC (\% $\mathrm{T}>\mathrm{MIC})$; (ii) the ratio of maximal drug concentration to MIC $\left(\mathrm{C}_{\max }: \mathrm{MIC}\right)$; and (iii) the ratio of the area under the concentrationtime curve at 24 hours to MIC (fAUC $\left.{ }_{0-24}: \mathrm{MIC}\right) .{ }^{[5]}$ The parameters of importance in determining efficacy as defined by the Pk-Pd target of an antimicrobial agent are therefore a function of drug exposure, which is largely determined by the dose administered and pharmacokinetics of the drug, and the MIC of the micro-organism. The clinical importance of this relationship has been demonstrated through correlation with clinical outcomes. The landmark DALI study ${ }^{[6]}$ showed that for betalactam agents, clinical outcome correlated with increasing $\% f \mathrm{~T}>\mathrm{MIC}$. The pivotal role of the MIC was further highlighted in this study, where a positive clinical outcome was 2.3 times more likely for pathogens with an MIC $\leq 2 \mu \mathrm{g} / \mathrm{mL}$. Knowledge of the MIC allows for more tailored antimicrobial therapy through improved choice of agent guided by an understanding of the pathogen and its MIC distribution, and (through application to simulated pharmacological data) the likely probability of Pk-Pd target attainment.

Despite the importance of the MIC, it is seldom considered in efforts to dose-optimise. This stems primarily from lack of awareness of its importance and knowledge of how to interpret MIC-based AST results, and the limited availability of this type of testing. We have developed capacity for gold-standard MIC-based AST that was driven largely by a need to compare generic with originator antimicrobial compounds. These comparative MIC studies are in vitro studies comparing the MIC of the originator with that of the generic against a collection of clinically relevant isolates using BMD, the current gold standard. Although relatively simple and devoid of significant scientific merit, the studies were born out of a need for justification, reassurance and quality control. It is reassuring to note that all generic manufacturers willing to subject their product to this comparative evaluation have demonstrated excellent in vitro microbiological efficacy and compliance with accepted concordance thresholds. More importantly, however, these studies have highlighted the importance of MIC testing. Table 1 provides an example of the valuable information provided by MIC testing. It highlights significant differences in ciprofloxacin susceptibility between two distinct phenotypic groups of Klebsiella pneumoniae isolates. Despite the equivalence in overall susceptibility rates, the MIC data demonstrate a marked elevation ( $>10$-fold) in MIC for ESBL-producing isolates. This has important implications for dose optimisation given the concentration-dependent nature of ciprofloxacin activity.

AMS is at present a global and national priority. It is considered a strategic objective of our national antimicrobial resistance framework and is a current metric in many hospitals. ${ }^{[7]}$ AMS is defined as 'coordinated interventions designed to improve and measure the appropriate use of antibiotic agents by promoting the selection of the optimal antibiotic drug regimen, including dosing, duration of therapy 
Table 1. Ciprofloxacin susceptibility of Klebsiella pneumoniae comparing the MIC values of ESBL-producing v. non-ESBLproducing isolates

\begin{tabular}{|c|c|c|c|}
\hline & $\operatorname{MIC50}(\mu \mathrm{g} / \mathrm{mL})$ & $\operatorname{MIC90}(\mu \mathrm{g} / \mathrm{mL})$ & Susceptible $^{\star}(\%)$ \\
\hline Non-ESBL-producing K. pneumoniae $(n=11)$ & $\leq 0.015$ & $\leq 0.015$ & 90.9 \\
\hline ESBL-producing K. pneumoniae $(n=35)$ & 0.25 & 0.5 & 90.9 \\
\hline
\end{tabular}

and route of administration. ${ }^{[8]}$ Dose optimisation is considered crucial, and an MIC (which I have already argued is a critical determinant of antimicrobial selection and dosing) therefore becomes synonymous with good AMS. Yet the role of laboratories in providing MIC data and MIC-based AST has largely been ignored and the majority of clinical decisions at the bedside rely solely on a categorical (S/I/R) interpretation. I do not advocate that treatment of every infectious disease process requires an MIC. This is too onerous for current laboratory capacity and is not necessary, as clinical breakpoints (by virtue of the '90/60 rule') allow for sufficient leeway. It does, however, require broad-scale consideration for when an MIC is required (at clinician and clinical microbiologist level) and how best to extract MIC-guided therapeutic decisions from currently employed AST methods (pathologist level).

Addressing the first requirement is relatively simple from an indication perspective, although widespread education is required to create awareness of this need. Any infectious disease where dose optimisation in the form of a tailored antimicrobial regimen is required would fulfil the criterion for MIC-based AST. This would include all significant infections in critically ill patients and the so-called 'difficultto-treat' infections, e.g. osteomyelitis, periprosthetic joint infections, infective endocarditis, etc. The second requirement is substantially more challenging for laboratories owing to limitations imposed by routine diagnostic AST systems (discussed above). Additional MIC testing of isolates against targeted antimicrobials could be performed based on predefined criteria such as source of specimen (e.g. blood) or location of patient (e.g. intensive care unit). This would require careful consideration by all stakeholders including policy-makers and funders. However, it will not account for all relevant scenarios, and close liaison between clinician and microbiologist would still be required to direct AST accordingly. A second possibility is to utilise current commercial systems based on more rigorous evaluation. The Vitek system (Biomerieux, South Africa (SA)) is currently used by the majority of diagnostic laboratories in SA and is capable of providing MICs. There is a concern among clinical microbiologists that these MICs (which are extrapolated from growth curves using sophisticated software) are not true MICs, and they have therefore not been reported routinely. There is some merit to this concern, although the requirements for approval by various regulatory authorities (including the US Food and Drug Administration) have been met and the system is therefore capable of providing MIC data that fulfil the essential agreement criteria (i.e. MIC within one two-fold dilution of the reference methodderived MIC). ${ }^{[9]}$ Importantly, any MIC value actually represents a range given the artificially created two-fold dilution scheme, and the precision associated with AST can vary by drug-bug combination. Through inter- and intra-laboratory comparative analysis utilising BMD and available MIC susceptibility data, it is possible to characterise isolates as being wild type (no acquired resistance mechanism) and to delineate the range of MICs based on specific phenotypes. The current Vitek AES software system already does this and many laboratories are reliant on it. This allows reporting of MICs with a sufficient degree of confidence and enables further directed MIC testing that is either guided clinically or based on a validated laboratory protocol. There is no one-size-fits-all approach to this methodology, so laboratories are required to evaluate their own isolates and systems. The advantage is that MIC-based AST becomes feasible in a routine diagnostic laboratory with provision of information that can better guide therapeutic decisions at the bedside.

The ensuing challenge is interpretation of MIC-based AST results, as we are accustomed to categorical interpretation only. The role of the clinical microbiologist becomes crucial in providing the necessary guidance in interpretation and selection of appropriate therapy. Clinical microbiologists themselves would need to become more familiar with MIC-based AST and the use of these data in guiding antimicrobial decisions. The information provided on microbiology reports should be more detailed, and we need to interrogate the reports with more vigour. The realisation that a simple AST categorisation may no longer suffice for each and every patient is a critical next step in ensuring dose optimisation. The role of MIC-based AST should be placed high on the agenda of AMS initiatives, with sustained education platforms aimed at addressing the current knowledge gap. Furthermore, there needs to be a focused drive by all laboratories to move to the EUCAST AST system following a 2015 national decision to adopt the EUCAST guidelines. The EUCAST guidelines provide robust, clinically relevant breakpoints supported by substantial MIC data that are freely available (www.eucast.org), thereby supporting and facilitating the implementation of MIC-based AST results.

The importance of the MIC in guiding therapeutic decisions for 'difficult-to-treat' infections cannot be overstated. The growing body of literature supports a move towards individualised and tailored antimicrobial therapy. ${ }^{[10]}$ This requires knowledge of the Pk-Pd parameters associated with clinical efficacy of an antimicrobial, which in turn creates a need for an MIC correlate given its direct relationship to these parameters. Furthermore, improved diagnostic determinants of drug exposure in each individual patient are required and this can only be attained through therapeutic drug monitoring (TDM). Only when armed with both an MIC and a drug level (TDM), taking into consideration the Pk-Pd correlate for a particular antimicrobial, will we truly be able to dose-optimise antimicrobial therapy and achieve the desired AMS outcomes.

Funding. GlaxoSmithKline Fellowship Grant and Aurobindo Pharma. Conflicts of interest. The author has received honoraria and funding support for MIC-based testing from the following pharmaceutical companies: Aspen, Aurobindo, MSD, Mylan, Pharmadynamics, Sandoz and Specpharm.

\section{Warren Lowman}

Department of Clinical Microbiology, Vermaak \& Partners/Pathcare Pathologists, Johannesburg, South Africa; Department of Clinical Microbiology and Infection Prevention and Control, Wits Donald Gordon Medical Centre, Johannesburg, South Africa; and Department of Clinical Microbiology and Infectious Diseases, Faculty of Health Sciences, University of the Witwatersrand, Johannesburg, South Africa warren.lowman@wits.ac.za 
1. EUCAST (European Committee on Antimicrobial Susceptibility Testing). Breakpoint tables for interpretation of MICs and zone diameters. 8.1, 2018. http://www.eucast.org (accessed 1 June 2018). 2. Rex JH, Pfaller MA. Has antifungal susceptibility testing come of age? Clin Infect Dis 2002;35(8):982989. https://doi.org/10.1086/342384

3. Mouton JW, Brown DF, Apfalter P, et al. The role of pharmacokinetics/pharmacodynamics in setting clinical MIC breakpoints: The EUCAST appro

4. Barbhaiya RH, Forgue ST, Gleason CR, et al. Pharmacokinetics of cefepime after single and multiple intravenous administrations in healthy subjects. Antimicrob Agents Chemother 1992;36(3):552-557. https://doi.org/10.1128/AAC.36.3.552

5. Ambrose PG, Bhavnani SM, Rubino CM, et al. Pharmacokinetics-pharmacodynamics of antimicrobial therapy: It's not just for mice anymore. Clin Infect Dis 2007;44(1):79-86. https://doi.org/10.1086/510079 . Roberts JA, Paul SK, Akova M, et al. DALI: Defining antibiotic levels in intensive care unit patients: Are current beta-lactam antibiotic doses sufficient for critically ill patients? Clin Infect Dis 2014;58(8): 10721083. https://doi.org/10.1093/cid/ciu02
7. Department of Health, South Africa. Antimicrobial Resistance National Strategy Framework, 2014 2024. http//wwwhealth govza/indexphp/component/phocadownload/category/199-antimicrobialresistance (accessed 1 June 2018).

8. Barlam TF, Cosgrove SE, Abbo LM, et al. Implementing an antibiotic stewardship program: Guidelines by the Infectious Diseases Society of America and the Society for Healthcare Epidemiology of America. Clin Infect Dis 2016;62(10):e51-e77. https://doi.org/10.1093/cid/ciw118

9. Jorgensen JH. Selection criteria for an antimicrobial susceptibility testing system. J Clin Microbiol 1993:31(11):2841-28n

10. Roberts JA, Abdul-Aziz MH, Lipman J, et al. Individualised antibiotic dosing for patients who are critically ill: Challenges and potential solutions. Lancet Infect Dis 2014;14(6):498-509. https://doi. org/10.1016/S1473-3099(14)70036-2

S Afr Med J 2018;108(9):710-712. DOI:10.7196/SAMJ.2018.v108i9.13285 